\title{
An Air Traffic Management Data Traffic Pattern for Aeronautical Communication System Evaluations
}

\author{
Thomas Gräupl, Nils Mäurer \\ Institute of Communications and Navigation \\ German Aerospace Center (DLR) \\ Wessling, Germany \\ thomas.graeupl@dlr.de,nils.maeurer@dlr.de
}

\begin{abstract}
Modern air traffic management relies on a communication infrastructure increasingly suffering from the VHF band's growing saturation in high density areas. Air traffic management communication shall therefore transition from analog VHF voice communication to more spectrum efficient digital data communication. These proposed digital technologies have to be evaluated carefully against the expected data traffic to ascertain that they offer the required capacity and performance. This paper describes the characteristics of air traffic management data and a method to generate a data traffic pattern suitable as input for the performance evaluation of digital aeronautical data communication systems. The data traffic pattern is derived from large scale computer simulations. Based on the simulation results, the paper provides guidance on how to model a data traffic pattern that is directly usable for practitioners wishing to evaluate the performance of aeronautical data communication systems.
\end{abstract}

Keywords - computer simulation; air traffic management; aeronautical communication.

\section{INTRODUCTION}

At the heart of the modern air traffic management system lays the communication, navigation and surveillance infrastructure enabling efficient aircraft guidance and safe separation in all phases of flight. However, although current systems are mature and generally providing good service, they are suffering from the VHF band's increasing saturation in high density areas. The EU and US, representing the two areas experiencing the most pressure, strive therefore for the sustainable modernization of the aeronautical communication infrastructure [1]. Air traffic management communication shall transition from ana$\log$ VHF voice communication to more spectrum efficient digital data communication supported by automated data processing and decision making of computer systems.

To enable the transition from analog to digital air traffic management communication, several data link systems are under development for the Future Communications Infrastructure (FCI). These proposed technologies have to be carefully evaluated against the expected future data traffic to ascertain that they will offer the required capacity and performance for future civil air traffic.

The aeronautical data traffic pattern has been examined in previous studies. Rokitansky et al. [2] provide statistics on the distribution of data message sizes in a proposed aeronautical satellite communication system. In his discussion of the optimization of TCP for aeronautical data traffic Ehammer et al. [3] examine Rokitansky's results in terms of the distribution of message sizes in relation to data traffic volume. Gräupl et al. [4] derive per-aircraft data rates and message rates for the dimensioning of another proposed aeronautical satellite system. Morlet et al. [5] continue this work providing additional statistics on message inter-arrival times. Ehammer et al. [6] applied this approach again to the performance evaluation of AeroMACS. All of the above studies rely on the "Communications Operating Concept and Requirements for the Future Radio System" developed by EUROCONTROL and FAA as source for future air traffic management services [7].

However, although conducted with data link performance evaluations in mind, none of the aforementioned studies provide clear guidance on a representative, practical, and quickly implementable data traffic pattern that can be used for the performance evaluation of aeronautical data links.

This paper describes the characteristics of air traffic management data and a method to generate traffic pattern suitable as input for the performance evaluation of aeronautical communication systems. The data traffic pattern is derived from large scale computer simulations modeling aeronautical data communication in great detail. Based on these results, the paper provides guidance on how to model a data traffic pattern that is immediately applicable for practitioners wishing to evaluate the performance of aeronautical data communication systems.

\section{BACKGROUND}

The results presented in this paper were developed for the flight trials of the L-band Digital Aeronautical Communication System (LDACS) [8] conducted in the MICONAV [9] project. The main objective of this development was to (1) define a data traffic pattern that is suitable to provide meaningful insight into the expected performance of LDACS and (2) practical to implement in flight trials.

A key problem when conducting flight trials of an aeronautical communication system is that one cannot rely on the communication system under test e.g. if one implements the measurement data traffic pattern as a true dialogue, where the answer to a message is triggered by a received message, a lost 
message may stop the measurement entirely. Thus we identified the following requirements:

- The data traffic pattern should be robust against partial communication failures: Lost messages should not cause the measurement to stop.

- The data traffic pattern should be implementable in a distributed environment with no or unreliable communication channels for coordination.

- The data traffic pattern should be realistic in terms of number, size, and arrival rate of packets at the radio.

\section{METHODS}

The results of this paper were generated using the Framework for Aeronautical Communications and Traffic Simulations 2 (FACTS2) [10]. FACTS2 is the next generation simulation framework designed and implemented by the German Aerospace Center (DLR). The FACTS2 simulation framework is a service oriented simulation framework. Particular simulations are implemented as toolchains of simulation services, implementing partial simulations of the overall simulation problem.

The simulation services employed in this paper are described in the following sections. On the highest level they can be grouped into services concerned with (1) air traffic simulation, and services concerned with (2) data traffic simulation.

\section{A. Air traffic simulation}

The air traffic simulation is based on the analysis of the IATA $^{1}$ database of worldwide scheduled passenger and cargo IFR flights for the years 2007 and 2008 [11]. On the basis of this database analysis we simulated a reference day of average European air traffic for the year 2007.

Flights are simulated individually. The simulation of each flight trajectory is implemented as a time-stepped simulation. Each flight is represented by a function $\mathrm{f}(\mathrm{t})$ returning its state vector as a function of time

$$
f(t)=s_{t} \text { for } \alpha \leq t \leq \omega
$$

where $\mathfrak{s}_{\mathrm{t}}$ is the state vector of the flight $\mathrm{f}$ at time $\mathrm{t} . \alpha$ is the start time of the flight, $\omega$ is the end time of the flight. $\mathfrak{s}_{t}$ comprises the position, altitude, air traffic control sector, and domain. An illustration of the flight state vector $\mathfrak{s}_{t}$ as a function of time $t$ is displayed in Figure 1.

For each hour of the reference day individual flights $f_{i}$ are generated such that the time between flight start times $\alpha_{i}$ is exponentially distributed according to the hourly flight generation rate extracted from the flight plan database. The flight generation rate is $1 /$ (inter-arrival time) according to Hoffmann et al. [13]. Flights $f_{i}$ are thus generated with exponentially distributed inter-arrival times such that $\alpha_{i+1}-\alpha_{i} \sim$ $\operatorname{Exp}\left(\gamma_{\mathrm{T}}(\mathrm{s}, \mathrm{d})\right) \forall \mathrm{s}, \mathrm{d} \in \mathfrak{A}$. Flight generation is thus a stationary

\footnotetext{
${ }^{1}$ The International Air Transport Association (IATA) is a trade association of
} the world's airlines.
Poisson process within each hour $\mathrm{T}$ where $\gamma_{T}(s, d)$ denotes the hourly flight generation rate for each pair of source and destination airports $s$ and $d$, and where $s, d \in \mathfrak{A}$, and $\mathfrak{A}$ is the set of airports in the flight plan database.

\section{B. Data Traffic Simulation}

The data traffic simulation is based on the Communications Operating Concept and Requirements for the Future Radio System (COCR) report [7]. COCR has been produced in a joint effort of EUROCONTROL and the Federal Aviation Administration (FAA) to identify concepts for future air traffic management communication. Its intended use is to help determining if candidate aeronautical communication technologies can meet the requirements of air traffic management.

COCR considers two phases of air traffic management communication. Phase I is based on existing data communication services. Phase II considers data communication as the primary means of air-ground communication supporting increased automation with new data link applications. Furthermore, COCR consider two types of data link applications: Addressed and broadcast applications. The broadcast services are surveillance services and direct air-to-air services that are not in the focus of air-ground data links.

This paper focuses on addressed phase II data link applications $^{2}$ to support the development of air-ground data links for the next decades.

COCR provides detailed descriptions for each data link application. The number and size of the messages to be exchanged between aircraft and ground is specified there. In addition, it is also defined whether the application is used periodically or triggered by events related to the progress of the flight.

Periodic applications are simulated by inserting the given number of data packets into the simulation result with the specified periodicity for each flight.

Event triggered applications are triggered by a change of air traffic control sector, a change of domain, a change of airport ramp position, or a change of flight phase as illustrated in Fig. 2. Trigger events thus correspond to changes in the flight state vector $f(t)=s_{t}$. The input to the simulation of these data link applications are therefore state changes of the flight state vector i.e. non-zero values of its derivative $\frac{d}{d t} f(t)$. The data load simulation requires the set of trigger events $\mathfrak{I}$ as input, which is

\footnotetext{
${ }^{2}$ We include the addressed COCR communication services for phase II: Air traffic services: ACL, ACM, AMC, ARMAND, COTRAC, D-ALERT, DATIS, DCL, D-FLUP, DLL, D-ORIS, D-OTIS, DRV-R, DSC, D-SIG, DSIGMET, D-TAXI, DYNAV, FLIPCY, FLIPINT, PPD, SAP, URCO; Airline operational communication: AOCDLL, CABINLOG, ENGINE, FLTLOG, FLTPLAN, FLTSTAT, FREETXT, FUEL, GATES, LOADSHT, MAINTPR, MAINTRT, NOTAM, OOOI, POSRPT, SWLOAD, TECHLOG, UPLIB, WXGRAPH, WXRT, WXTEXT; Network services: NETCONN, NETKEEP. The broadcast services are not included: A-EXEC, AIRSEP SURV, C\&P SURV, ITP SURV, M\&S SURV, PAIRAPP SURV, C\&P ACL, ITP ACL, PAIRAPP ACL, M\&S ACL, SURV ATC, WAKE.
} 


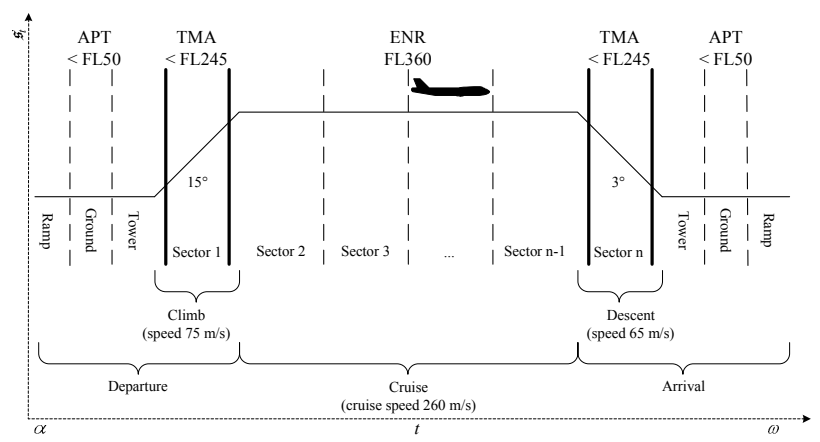

Figure 1: Illustration of the flight state vector $\mathfrak{s}_{t}$ as function of time $t$. The position component of the state vector $\mathfrak{s}_{t}$ is not displayed.

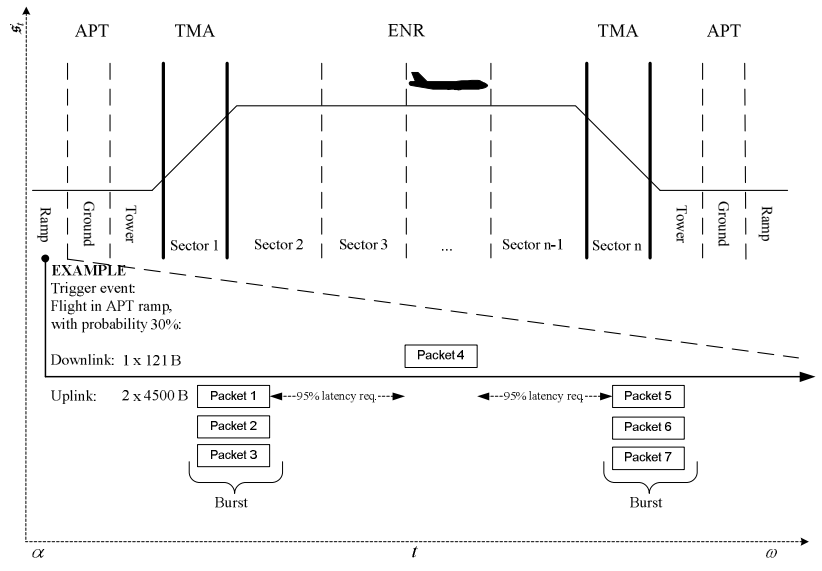

Figure 2: Illustration of the data traffic simulation as a function of the flight state vector $\mathfrak{s}_{t}=f(t)$. Non-zero values of $\frac{d}{d t} f(t)$ are matched against the trigger events defined for each service in COCR. If the values match the simulation of the data traffic service is triggered with the given probability. Uplink and downlink packets are then exchanged separated by the $95 \%$ percentile of the required service latency. Application layer messages larger than the Ethernet MTU size are split into bursts of MTU-sized packets.

$$
\mathfrak{T}=\left\{\left(t, \frac{d}{d t} f_{i, s, d}(t)\right) \mid \frac{d}{d t} f_{i, s, d}(t) \neq 0\right\}
$$

Thus the implementation of the data traffic simulation can be reduced to an event driven simulation on the basis of the trigger events received through the simulation toolchain interface: On the reception of each simulation event, the number of data packets specified in COCR for this event are added to the simulation output. Data packets are separated in time by the specified $95 \%$ percentile latency requirement of the applica- tion. This approach is illustrated in Figure 2 for an example service.

In order to simulate a realistic distribution of packet sizes at the air-ground data link large application packets are split into bursts of smaller packets in the simulation. We assume that a future air-ground data link radio would be connected to an Ethernet-like local area network in the aircraft (e.g. AFDX) and on the ground. Large application packets would therefore be split into bursts of packets not larger than the Ethernet MTU size.

Some event triggered applications need to be invoked several times until the flight changes its state again. For the uniform distribution of such communication exchanges it is necessary to know how long the flight will remain in the relevant state. This information is available through the individual simulation of the flight trajectories.

\section{RESULT}

The characteristics of air traffic management data traffic are derived from the result of the computer simulation described in the last section.

The characteristics discussed in this paper are per-flight characteristics and not aggregate characteristics of the complete simulation scenario i.e. continental Europe. The detailed results of the computer simulation for the European Statistical Reference Area (ESRA08) are discussed in [10]. The overall simulation scenario is, however, not relevant in the context of this paper if the following points are taken into account:

(1) The simulation scenario must be large enough to provide a good sample of individual flights for the characterization of per-flight data traffic patterns. This is the case in our study because the simulation comprises 27,302 IFR flights with a peak instantaneous aircraft count of 3,579 aircraft. It is therefore not necessary to extrapolate the simulation scenario to future higher air traffic scenarios as it has been done in [10] since the sample is large enough for per-aircraft characteristics.

(2) The simulation scenario must take the correct data traffic pattern into account. This is the case because we simulate the COCR phase II data link services modeling the air traffic management applications of the coming decades. Thus, since we do not take surveillance broadcast services into account, the data traffic pattern does not depend on the number of flights in the simulation, too.

\section{A. Per-flight data rate}

The average data rate per aircraft is $291.44 \mathrm{bit} / \mathrm{s}$ from ground to air and $80.03 \mathrm{bit} / \mathrm{s}$ from air to ground.

The $99 \%$ percentile, which is often used to characterize the peak data rate, is $1,056.0 \mathrm{bit} / \mathrm{s}$ from ground to air and 856.0 $\mathrm{bit} / \mathrm{s}$ from air to ground, respectively.

This indicates that the average data rate for ground to air communication is significantly (3.64 times) larger than the average data rate in the reverse direction. The peak data rates are, however, comparable and in the order of $1 \mathrm{kbit} / \mathrm{s}$. 


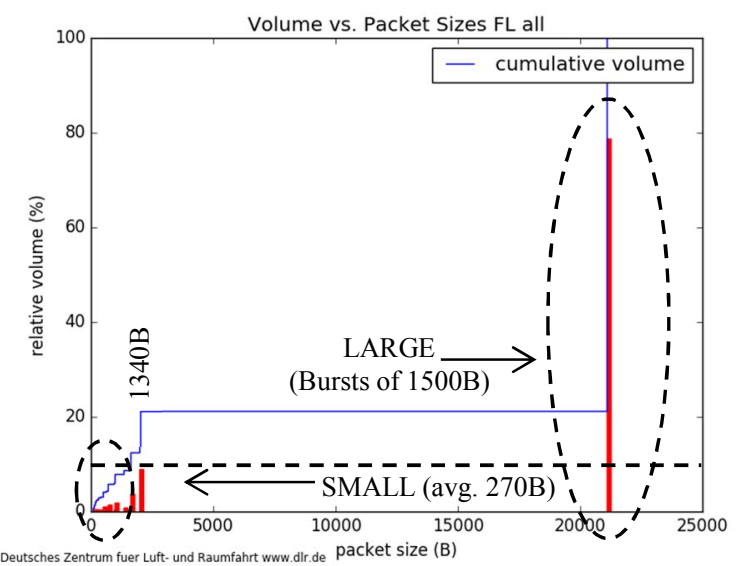

Figure 3: Relative and cumulative communication volume in the ground-to-air direction (FL; forward link) distributed according to packet size. Note that approximately $9 \%$ of the communication volume is generated by "small" packets and approximately $91 \%$ of the communication volume is generated by bursts of "large" packets. Note that packets of size $1340 \mathrm{~B}$ are regarded as "large" packets although they are not marked as such in the figure.

\section{B. Distribution of packet size}

It is instructive to look at the size of the packets generating the data traffic rates indicated above. There is a clear dichotomy between "small" and "large" packets visible in Figure 3 (ground-to-air) and Figure 5 (air-to-ground).

\section{1) Ground to air communication}

In the ground-to-air direction $73.78 \%$ of packets are smaller than $1340 \mathrm{~B}$ as indicated by the jump of the cumulative volume in Figure 3 . They generate $8.66 \%$ of the communication volume as shown in Figure 4 . The weighted average size of the "small" packets is $270 \mathrm{~B}$.

$26.22 \%$ of packets are "large" packets larger than $1340 \mathrm{~B}$. They generate $91.34 \%$ of the communication volume. However, it should be noted that $78.65 \%$ of the volume is generated by application layer messages larger than the Ethernet MTU size. That is, this part of the data traffic volume is generated by bursts of MTU sized packets, because these packets are fragmented in multiple MTU-sized packets. Packets of 1500B size are therefore the most frequent "large" packets.

Inspecting the services generating these packets reveals that the "small" packets are generated by Air Traffic Services (ATS) applications and the "large" packets are generated by Airline Operational Communication (AOC) services. Although both ATS and AOC are regarded as being related to the safety and the regularity of the flight, ATS services have clear priority over AOC services.

It should be noted that the generation of large packets is dominated by a single AOC service. $79 \%$ of the total data traffic of phase II communication is contributed by the graphical weather service (WXGRAPH).

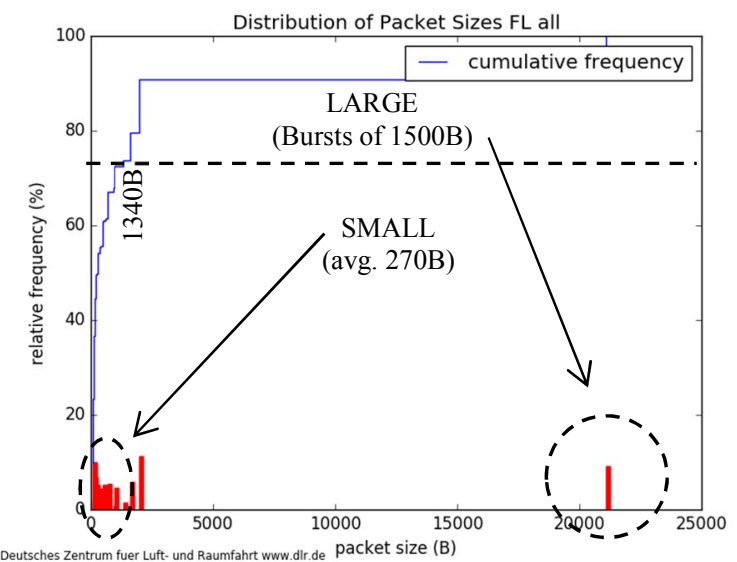

Figure 4: Relative and cumulative frequency of packet sizes in ground-to-air direction (FL; forward link). Note that approximately $74 \%$ of all packets are "small" packets and approximately $26 \%$ of all packets are bursts of "large" packets. Note that packets of size 1340 B are regarded as "large" packets although they are not marked as such in the figure.

\section{2) Air to ground communication}

In the air-to-ground direction the split between large and small packets is even clearer visible in Figure 5. In the data traffic originating from the aircraft $29.68 \%$ of the communication volume is generated by packets smaller than $1380 \mathrm{~B}$ as indicated by the jump of the cumulative volume graph. The remaining $70.32 \%$ of communication volume is generated by packets larger than 1380B.

If we inspect the frequency of packet sizes in Figure 6 we can see that the weighted average size of the "small" packets is 121B. Most of the "large" packets have size 1380 B (79.40\% of the large packets). Only a few "large" packets are larger than the MTU size and produce occasional bursts.

The air-to-ground direction is thus dominated by "small" (121B) and "large" (1380B) packets as indicated in Figure 6. The split of "small" packets being generated by ATS services and "large" packets being generated by AOC services can also be observed here.

It should be noted that air to ground communication is not dominated by a single service like the ground to air communication is dominated by the graphical weather service.

\section{DISCUSSION}

Our air-to-ground results are similar to the results reported by Ehammer [3], who reported approximately $70 \%$ of the RL packets to be smaller than $175 \mathrm{~B}$ and approximately $30 \%$ of the RL packets to be larger than $1400 \mathrm{~B}$. The ground-to-air result is not directly comparable, since Ehammer et al. did not take the segmentation of packets larger than the Ethernet MTU into account in their analysis of the distribution of packet sizes. However, Ehammer's overall summary still holds: The aeronautical data traffic pattern generates either bursts of large packets (generating most of the traffic volume) or single small packets (generating most of the packets). 


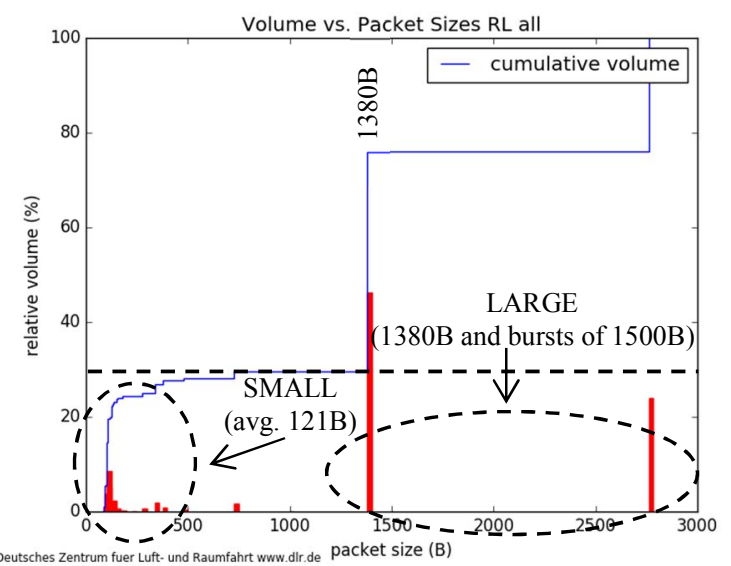

Figure 5: Relative and cumulative communication volume in the air-to-ground direction (RL; reverse link) distributed according to packet size. Note that approximately $30 \%$ of the communication volume is generated by "small" packets and approximately $70 \%$ of the communication volume is generated by "large" packets.

We can add in this study, that burst of large packets are more common in the ground-to-air direction, which is also reflected in the significantly higher standard deviation of its data rate. The $99 \%$ percentile of the data rate is, however, comparable both in the ground-to-air direction (1056 bit/s) and the air-to-ground direction ( $856 \mathrm{bit} / \mathrm{s})$, indicating that a future aeronautical data link design should be able to deal with similar peak data rates in both directions.

\section{A. Guidance on generating air traffic management data traffic for performance evaluation}

In order to support the development of robust future aeronautical data links we recommend designing the data link to be able to cope with the peak data rates identified in this paper. Our recommendation is that $1 \mathrm{kbit} / \mathrm{s}$ should be used as an estimate of the per-aircraft data rate in the ground-to-air direction, and $0.85 \mathrm{kbit} / \mathrm{s}$ in the air-to-ground direction. This is larger than the $99 \%$ percentile of the average data rate and provides therefore a good upper bound for the worst case.

\section{1) Ground to air data traffic pattern}

In the ground-to-air direction the distribution of packet sizes should approximate the most frequent packet sizes observed in the data traffic pattern: $75 \%$ packets with size 270 Bytes and $25 \%$ bursts of 3 MTU sized $(1,500 \mathrm{~B})$ packets. This results in a realistic data traffic pattern where $10 \%$ of the data traffic volume is generated by "small" packets and $90 \%$ of the volume is generated by bursts of "large" packets.

We assume that very large application layer messages would be transmitted via TCP. TCP opens the sending window linearly. Thus, transmitting 14 packets (equivalent to one WXGRAPH application layer message) would result in an average sending window size of 3 packets and a maximum sending window size of 4 packets. We recommend therefore generating burst of 3 packets to model this behavior.

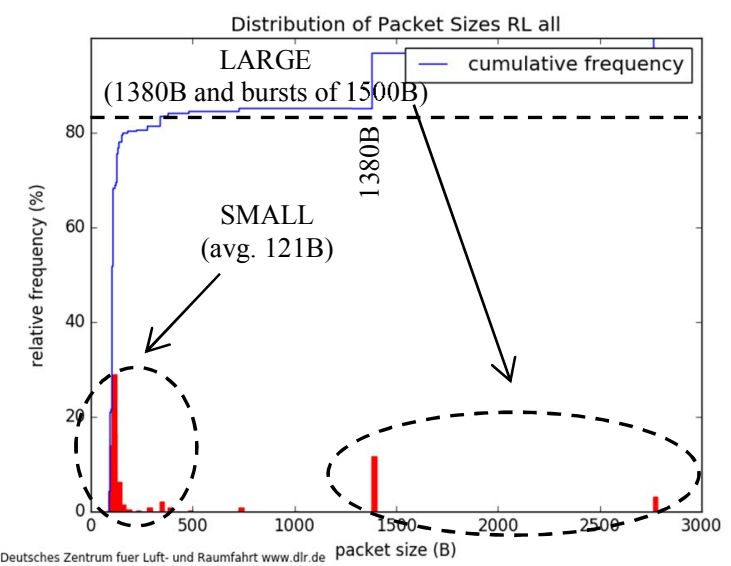

Figure 6: Relative and cumulative frequency of packet sizes in air-to-ground direction (RL; reverse link). Note that approximately $80 \%$ of all packets are "small" packets and approximately $20 \%$ of all packets are "large" packets.

"Small" packets should have priority over "large" packets in order to model the priority of ATS data traffic over AOC data traffic.

\section{2) Air to ground data traffic pattern}

In the air-to-ground direction the distribution of packet sizes should approximate the most frequent packet sizes observed in the data traffic pattern: $80 \%$ packets with size 121 Bytes and $20 \%$ packets with size 1380 Bytes. This results in a realistic data traffic pattern where $30 \%$ of the data traffic volume is generated by "small" packets and $70 \%$ of the volume is generated by "large" packets.

"Small" packets should have priority over "large" packets in order to model the priority of ATS data traffic over AOC data traffic.

We recommend to model the packet generation process as a Poisson process i.e. we recommend to use exponentially distributed inter-packet arrival times. The parameter of the exponential distribution can be computed as follows: The expected value of an exponentially distributed random variable $\mathrm{X}$ is given by

$$
E[X]=\frac{1}{\lambda}
$$

where $\lambda$ is the only parameter of the exponential distribution. This means that $\lambda$ has to be set to the multiplicative inverse of the desired mean packet inter-arrival time. In our case this is equal to the mean time interval between packets which can be calculated as the inverse of the mean number of packet per time unit, which is the quotient of the average data rate over the average packet/burst size. For the ground-to-air direction this amounts to

$$
\lambda=\frac{1 \text { kbit }}{0.75 \cdot 270 B+0.25 \cdot 3 \cdot 1500 B}=\frac{1}{10.62} .
$$


Table 1: Recommendation on generating per-aircraft ATM and AOC data traffic for performance evaluation.

\begin{tabular}{|c|c|c|c|}
\hline Per Aircraft & Ground to air & Air to ground & Priority \\
\hline Data rate & $1 \mathrm{kbit} / \mathrm{s}$ & $0.85 \mathrm{kbit} / \mathrm{s}$ & \\
\hline Packet/burst & $75 \%: 270 \mathrm{~B}$ & $80 \%: 121 \mathrm{~B}$ & $\begin{array}{l}\text { High } \\
\text { (ATS) }\end{array}$ \\
\hline $\begin{array}{r}\text { size distribu- } \\
\text { tion }\end{array}$ & $25 \%: 3 \times 1500 \mathrm{~B}$ & 20\%: $1380 \mathrm{~B}$ & $\begin{array}{l}\text { Low } \\
\text { (AOC) }\end{array}$ \\
\hline $\begin{array}{c}\text { Packet/burst } \\
\text { inter-arrival } \\
\text { time }(1 / \lambda)\end{array}$ & $10.62 \mathrm{~s}$ & $3.50 \mathrm{~s}$ & \\
\hline
\end{tabular}

The mean packet inter arrival time for the ground-to-air direction is therefore 10.62 seconds per aircraft and the parameter of the exponential distribution $\lambda=\frac{1}{10.62}$. A similar calculation can be made for the air-to-ground direction.

Note that packet bursts need not be modeled in the air-toground direction since there is only one service with overlarge packets (FLIPINT has packets of size 2763 B) and low occurrence.

Table 1 summarizes the recommendations of this section. Note that these numbers should be applied per-aircraft. That is, if $n$ aircraft should be evaluated, this model should be applied $n$ times, thus once for each aircraft. $n$ should be small to avoid overestimating the aggregate traffic. For large $n$ the aggregate data rates provided in COCR should be used.

\section{LIMITATIONS OF THIS WORK}

The authors of this work are aware that updated versions of the COCR data traffic pattern exist. However, none of these data traffic patterns is publicly available since they are mostly based on proprietary data provided by service providers.These models could therefore not be taken into account in this study.

\section{CONCLUSION}

The objective of this paper was to provide guidance on how to model a data traffic pattern that is immediately usable for practitioners wishing to evaluate the performance of aeronautical data communication systems.

We have presented these recommendations in this paper together with a practical method usable for anyone wishing to evaluate the performance of aeronautical data communication systems. We derive this data traffic pattern from the characterization and the computer simulation discussed in the previous chapters. However, it should be noted that the goal of our guidance was not to generate data traffic patterns mirroring the presented results exactly. The presented results build on assumptions made in the COCR report and in the implementation and evaluation of our computer simulation in the FACTS2 framework. The resulting characterization should therefore be assumed to echo these assumptions as well and thus be treated with care. Although we do not think that our simulation results (or indeed any simulation results) can predict the future of aeronautical communication exactly, we do think, however, that our results are suitable to derive robust guidance on modeling aeronautical data traffic for the evaluation of aeronautical data link prototypes.

\section{REFERENCES}

EUROCONTROL, "Challenges of Growth 2013 - Task 4: European Air Traffic in 2035," 2013. [Online]. Available: http://www.eurocontrol.int/articles/challenges-growth.

C.-H. C.-H. Rokitansky, M. Ehammer, and T. Gräupl, "Communication capacity assesment for the Iris satellite system," in Digital Avionics Systems Conference, 2008. DASC 2008. IEEE/AIAA 27th, 2008, p. 2--B.

[3] M. Ehammer, T. Gräupl, and C.-H. H. Rokitansky, "TCP/IP over aeronautical communication systems - Effects on bandwidth consumption," in AIAA/IEEE Digital Avionics Systems Conference Proceedings, 2009.

T. Gräupl and T. Gräupl, "Dimensioning requirements for the ANTARES ATM satellite data-link," in Integrated Communications Navigation and Surveillance Conference (ICNS), 2010, 2010, pp. 122.

[5] C. Morlet, M. Ehammer, T. Gräupl, and C.-H. H. Rokitansky, "Characterisation of the data link communication air traffic for the European airspace," in AIAA/IEEE Digital Avionics Systems Conference - Proceedings, 2010.

[6] M. Ehammer, T. Graupl, and E. Polo, "AeroMACS data traffic model," 2011 Integr. Commun. Navig. Surveill. Conf. Proc., pp. G51-G5-10, 2011.

[7] Eurocontrol and FAA, "Communications Operating concept and Requirements for the Future Radio System (COCR)," Eurocontrol/FAA, 2007.

[8] M. Schnell, U. Epple, D. Shutin, and N. Schneckenburger, "LDACS: future aeronautical communications for air-traffic management," Commun. Mag. IEEE, vol. 52, no. 5, pp. 104-110, 2014.

[9] T. Gräupl, N. Schneckenburger, T. Jost, M. Schnell, A. Filip, M. A. Bellido-Manganell, D. M. Mielke, N. Mäurer, R. Kumar, O. Osechas, G. Battista, T. Bögl, and T. Richter, "L-band digital aeronautical communications system flight trials in the national German project MICONAV," in ICNS 2018 - Integrated Communications, Navigation, Surveillance Conference, 2018.

[10] T. Gräupl, "FACTS2: Extended simulation framework for ATM communication demand analysis of Europe," in AIAA/IEEE Digital Avionics Systems Conference - Proceedings, 2017.

[11] F. Hoffmann, U. Epple, M. Schnell, and U. Fiebig, "Feasibility of LDACS1 cell planning in European airspace," in Digital Avionics Systems Conference (DASC), 2012 IEEE/AIAA 31st, 2012, p. 5E1-15E1-13.

[12] EUROCONTROL, "Long-Term Forecast: IFR Flight Movements 2010-2030," $2010 . \quad$ [Online]. Available: http://www.eurocontrol.int/articles/challenges-growth.

[13] F. Hoffmann, C. Bauer, D. Medina, and S. Ayaz, "FACTS: An OMNeT++ Based Simulator for Aeronautical Communications," in Proceedings of the 1st International Conference on Simulation Tools and Techniques for Communications, Networks and Systems \& Workshops, 2008, p. 85:1--85:4.

[14] EUROCONTROL and FAA, "Communication Operating Concept and Requirements for the Future Radio System, Ver. 2.0,” 2007. 